\title{
RX J2217.9-5941: A highly X-ray variable Narrow-Line Seyfert1 galaxy ${ }^{\star}$
}

\author{
D. Grupe ${ }^{1}$, H.-C. Thomas ${ }^{2}$, and K. M. Leighly ${ }^{3,4}$ \\ 1 MPI für extraterrestrische Physik, Postfach 1312, 85741 Garching, Germany \\ 2 MPI für Astrophysik, Karl-Schwarzschild-Str. 1, 85748 Garching, Germany \\ 3 Columbia Astrophysics Laboratory, 538 West 120th St., New York, NY 10027, USA \\ 4 Dept. of Physics and Astronomy, University of Oklahoma, 440 W. Brooks St., Norman, OK 73019, USA
}

Received 26 October 2000 / Accepted 12 January 2001

\begin{abstract}
We report the discovery of a highly X-ray variable AGN, RX J2217.9-5941. This object was bright during the ROSAT All-Sky Survey (RASS), during which a decrease in the count rate by a factor of 12 was observed. It was found to be much fainter in follow-up HRI observations and is therefore an X-ray transient AGN candidate. On long time scales of years, its count rate decreased by a factor of about 30 between the RASS and the ROSAT HRI and ASCA observations in 1998. Analysis of the ASCA data, complicated by source confusion and poor statistics, tentatively indicates a steep spectrum in the faint state. There is no evidence for variability among 5 optical observations of the object obtained between 1992 and 1998. We discuss the variability of RX J2217.9-5941 and its possible X-ray transient nature.
\end{abstract}

Key words. accretion, accretion disks - galaxies: active - galaxies: nuclei - galaxies: Seyfert - galaxies: individual (RX J2217.9-5941)

\section{Introduction}

With the launch of the X-ray satellite ROSAT (Trümper 1983) it had become possible to study the X-ray spectra of Active Galactic Nuclei (AGN) in the energy band between 0.1 and $2.4 \mathrm{keV}$ with the Position Sensitive Proportional Counter (PSPC, Pfeffermann et al. 1986). In this energy band AGN show typical variabilities by factors of $2-3$ on timescales of years (e.g. Grupe et al. 2001). However, some AGN show rapid X-ray variabilities by factors of more than a factor of ten on time scales of days. The most extreme example of these persistent rapid X-ray variable AGN is the Narrow-line Seyfert 1 galaxy (NLS1) IRAS 13224-3809 which has shown flux variabilities of almost 60 in only two days and a shortest doubling timescale of $800 \mathrm{~s}$ (Boller et al. 1997a). Another example is the NLS1 PHL 1092 which has shown a maximum intensity variability by a factor of $\approx 21$ between Einstein and ROSAT observations (Forster \& Halpern 1996) and by a factor of $\approx 14$ during ROSAT High Resolution Imager (HRI) monitoring (Brandt et al. 1999).

\footnotetext{
Send offprint requests to: D. Grupe, e-mail: dgrupe@xray.mpe.mpg.de

* Bases in part on observations at the European Southern Observatory La Silla (Chile) with the ESO/MPG $2.2 \mathrm{~m}$ telescope, the ESO $1.52 \mathrm{~m}$ telescope in August 1992 and September 1995, and the ESO $3.6 \mathrm{~m}$ in January 2001.
}

The most extreme form of variability is the X-ray transience in AGN, a new type of AGN phenomenon that has been established by the ROSAT All-Sky Survey (RASS, Voges et al. 1999). X-ray transient AGN are typically identified as bright sources in their "high" state phase during the RASS, and are then found to be dramatically fainter or even to have disappeared by the time of follow-up pointed observations years later. Some prominent examples of ROSAT discovered transients are IC 3599 (Brandt et al. 1995; Grupe et al. 1995a), WPVS007 (Grupe et al. 1995b), or RX J0134.2-4258 (Grupe et al. 2000). X-ray transience has even been discovered in nonactive galaxies such as RX J1624.9+7554 (Grupe et al. 1999b), RX J1242.6-1119 (Komossa \& Greiner 1999), or RX J1420.4+5334 (Greiner et al. 2000).

X-ray variability in AGN can be explained by either intrinsic variability plausibly caused by such factors as changes in the accretion rate or relativistic boosting effects (e.g. Boller et al. 1997a) or by changes of the cold and warm absorption column densities towards the source (e.g. Abrassart \& Czerny 2000; Schartel et al. 1997; Komossa \& Meerschweinchen 2000).

We performed a variability study of a complete sample of bright soft X-ray selected AGN (Grupe et al. 2001) and found RX J2217.9-5142 to be one of the most variable sources on short as well as long timescales. Optically, we identified RX J2217.9-5142 as a NLS1 with $F W H M(\mathrm{H} \beta)=1850 \mathrm{~km} \mathrm{~s}^{-1}$ and very strong FeII emission at a redshift of $z=0.160$ (Grupe et al. 1999a). 
The paper is organized as follows: Sect. 2 describes the X-ray and optical observations, Sect. 3 presents the results which are discussed in Sect. 4. Throughout the paper, luminosities are calculated assuming a Hubble constant of $H_{0}=75 \mathrm{~km} \mathrm{~s}^{-1} \mathrm{Mpc}^{-1}$ and a deceleration parameter of $q_{0}=0$, except if noted otherwise. Spectral slopes, $\alpha$, are defined by $F \propto \nu^{-\alpha}$.

\section{Observations and data reduction}

RX J2217.9-5941 was observed during the RASS between 22 October 1990, 12:32 and 24 October 1990, 19:00 for a total of $587 \mathrm{~s}$. We used the photon event file of the RASS II processing. Photons were extracted in a circle of $250^{\prime \prime}$ radius for the source and two circles of $400^{\prime \prime}$ in scan direction for the background. RX J2217.9-5941 was observed twice by the ROSAT HRI detector: 1) ROR 702894, 06 May 1997 13:09:44 - 10 May 1997 02:12:42 for a total exosure of $4476 \mathrm{~s}$, and 2) ROR 601124, 15 April 1998 16:32:36 - 18 April 1998 00:42:42 for a total of 9760 s. Source photons were collected in a circle of $40^{\prime \prime}$ radius and background in a ring with radii of $50^{\prime \prime}-150^{\prime \prime}$ around the source.

ASCA observed RX J2217.9-5941 on 5 May 1998. Standard processing yielded a total exposure in the SIS detectors of approximately $40 \mathrm{ks}$. RX J2217.9-5941 was in a low state during the observation, and analysis was complicated by the fact that two sources, both approximately 4 arcmin away and both detected in the $H R I$ image (Fig. 1), were rather bright during the observation. These objects are identified as RX J2217.9-5937 and RX J2218.0-5939 respectively (sources B and C in Fig. 1). Thus, care was required for imaging and spectral analysis. Therefore we discarded a period of about $10.5 \mathrm{ks}$ at the beginning of the observation that was characterized by somewhat unstable pointing (although within the standard limits) and a very bright background flare of unknown origin that was inexplicably not detected by the usual background monitors. The final usable exposure was $29.5 \mathrm{ks}$.

$V$ and $B$ images with exposure times of 5 and $10 \mathrm{~min}$ were taken during an identification run for ROSAT discovered X-ray sources (Thomas et al. 1998) on 18th August 1992 with the ESO/MPI $2.2 \mathrm{~m}$ telescope at La Silla. Optical spectra were taken in 1992 and 1993 with the ESO/MPG $2.2 \mathrm{~m}$ telescope, in 1995 with the ESO $1.52 \mathrm{~m}$, and with the $1.5 \mathrm{~m}$ Ritchey-Chretien telescope at CTIO in 1998 . We note that the 1998 observation was made with accurate spectrophotometry in mind; therefore a $4.5^{\prime \prime}$ slit was used under photometric conditions and all of the light from the AGN was extracted during the reductions.

Data reduction was performed with EXSAS (Zimmermann et al. 1998) for the ROSAT data, MIDAS for the optical data (except for the 1998 spectrum) and XSELECT for the ASCA data. ROSAT HRI and ASCA count rates are converted into ROSAT PSPC count rates using the W3PIMMS program of NASA's Goddard Space Flight Center (version 2.7, 1999, http://heasarc.gsfc.nasa.gov/Tools/w3pimms.html) assuming no spectral changes between the RASS and the pointed observations, and based on the power-law fit to the RASS data with the column density $N_{\mathrm{H}}$ fixed to the Galactic value of Dickey \& Lockman (1990; $N_{\mathrm{H}}=2.5810^{20} \mathrm{~cm}^{-2}$ ).

Infrared data where taken from the Infrared Processing and Analysis Center (IPAC) using the interactive xscampi program.

\section{Results}

\subsection{Identification security}

The X-ray position from the RASS is $\alpha_{2000}=$ $22^{\mathrm{h}} 17^{\mathrm{m}} 57.1^{\mathrm{s}}, \delta_{2000}=-59^{\circ} 41^{\prime} 34.0^{\prime \prime}$ with a $2 \sigma$ error radius of $11.5^{\prime \prime}$. Figure 2 displays the $V$ image of RX J2217.95941 and the error radius around the RASS position of the source. Another source is present inside the $90 \%$ confidence circle (\#2 in Fig. 2). There are a couple of pieces of evidence that \#1 is RX J2217.9-5941: 1) The position of the bright source in the center of the $H R I$ observation is $\alpha_{2000}=22^{\mathrm{h}} 17^{\mathrm{m}} 56.7, \delta_{2000}=-59^{\circ} 41^{\prime} 31.2^{\prime \prime}$, only $1.8^{\prime \prime}$ away from source \#1 in Fig. 2 (see Table 1); 2) the identification spectrum shows a typical NLS1, which often have very steep X-ray spectra (see Boller et al. 1996; Grupe et al. 1998a, 1999a); 3) It is the bluest object among all four bright sources in the field (see Table 1), and 4) the optical spectrum of source \#2 shows that of a K-star. Using the statistic for the ratio of the X-ray to optical luminosity of K-stars (Beuermann et al. 1999) we expect source \#2 to have a count rate of $0.002 \mathrm{cts} \mathrm{s}^{-1}$. A $1 \sigma$ deviation from the average would result in a count rate of $0.007 \mathrm{cts} \mathrm{s}^{-1}$. So its contribution to the X-ray luminosity of RX J2217.9-5941 is expected to be negligible.

Figure 1 displays the HRI image of the surroundings of RX J2217.9-5941. The image was created from the merged event file of both $H R I$ observations in order to improve the signal-to-noise ratio in that image. The sources A, B, and $\mathrm{C}$ refer to the definition given in Table 2. Source $\mathrm{A}$ is identical with our AGN RX J2217.9-5941 and the HRI position is given in the paragraph above. Source B has the $H R I$ position $\alpha_{2000}=22^{\mathrm{h}} 17^{\mathrm{m}} 55.3, \delta_{2000}=-59^{\circ} 37^{\prime} 39.2^{\prime \prime}$ and source $\mathrm{C} \alpha_{2000}=22^{\mathrm{h}} 18^{\mathrm{m}} 03.2, \delta_{2000}=-59^{\circ} 39^{\prime} 15.2^{\prime \prime}$. Both sources are close to the edge of the extraction radius of 250" of the RASS data of RX J2217.9-5941 (source A), but any contribution of these sources can be neglegted.

\subsection{X-rays}

\subsubsection{ROSAT observations}

We found that RX J2217.9-5941 suffered a decrease in its count rate by a factor of about 30 between its RASS coverage and the HRI observation in 1998. During the RASS observation RX J2217.9-5941 had a count rate of $0.83 \pm 0.06 \mathrm{cts} \mathrm{s}^{-1}$ (Thomas et al. 1998). The HRI pointings result in $0.0519 \pm 0.0045 H R I \mathrm{cts} \mathrm{s}^{-1}$ for the 1997 observation and $0.0054 \pm 0.0018 H R I \mathrm{cts} \mathrm{s}^{-1}$ for the 1998 observation, which correspond to 0.256 and $0.026 \mathrm{PSPC}_{\mathrm{cts} \mathrm{s}}{ }^{-1}$ using W3PIMMS, respectively (see Sect. 2). 
Table 1. Summary of the sources \#1-\#4 in Fig. 2 Coordinates are from the US Naval Observatory A2 scans, $b$ and $r$ are the magnitudes measured from the USNO scans, $B_{\mathrm{j}}$ and $O R$ from the APM scans, and $B, V$, and $B-V$ from the measurements of our $B$ and $V$ images

\begin{tabular}{|c|c|c|c|c|c|c|c|c|c|}
\hline \multirow[b]{2}{*}{$\#$} & \multirow[b]{2}{*}{$\alpha_{2000}$} & \multirow{2}{*}{$\delta_{2000}$} & \multicolumn{2}{|c|}{ USNO A2 } & \multicolumn{2}{|c|}{ APM mag } & \multicolumn{3}{|c|}{ ESO images } \\
\hline & & & $b$ & $r$ & $B_{\mathrm{j}}$ & $O R$ & $B$ & V & $B-V$ \\
\hline 1 & 221756.61 & -594130.2 & 16.4 & 15.9 & 15.73 & 15.36 & 16.88 & 16.54 & 0.34 \\
\hline 2 & 221756.06 & -594141.4 & 17.3 & 15.4 & 16.17 & 14.90 & 16.98 & 16.13 & 0.85 \\
\hline 3 & 221756.90 & -594200.5 & 17.2 & 15.6 & 15.95 & 15.18 & 17.24 & 16.52 & 0.72 \\
\hline 4 & 221758.18 & -594110.0 & 17.0 & 15.4 & 16.89 & 15.69 & 18.64 & 17.65 & 0.99 \\
\hline
\end{tabular}

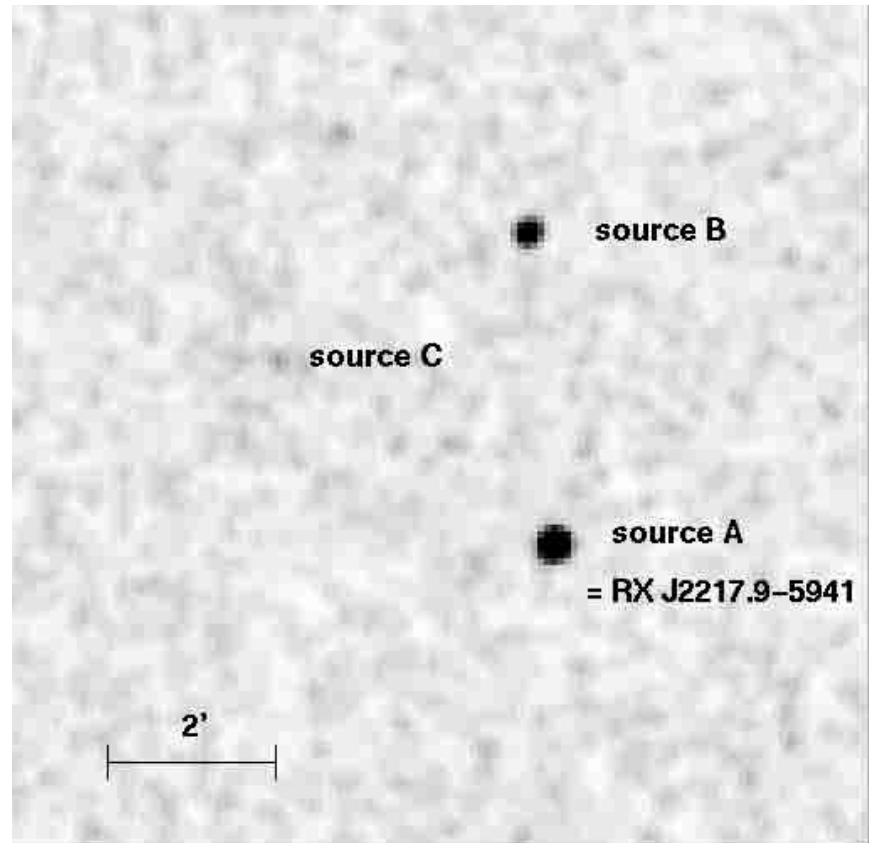

Fig. 1. HRI image of RX J2217.9-5941. The sources A, B, C refere to the definitions given in Table 2. See also Sect. 3.1 for the exact $H R I$ positions of those sources

We performed standard power-law spectral fits to the RASS spectrum, with all parameters free or with neutral absorption fixed to the Galactic value. The results of the single power-law fits to the RASS spectra are listed in Grupe et al. (2001). The best fit was obtained when the column density was free to vary; however, this results in less absorption than what is predicted from the Dickey \& Lockman maps (1990). We infer this to be evidence for a soft excess in the spectrum. When the column density is fixed to the Galactic value, we derived a spectral slope of $\alpha_{\mathrm{x}}=2.7$, one of the steepest among the AGN in our soft X-ray selected AGN sample (Grupe et al. 2001). The restframe luminosity in the ROSAT band is $\log L_{0.2-2.0}=$ $37.5[\mathrm{~W}]$. Due to the small number of collected photons during the RASS coverage complicated spectral fits were not warranted.

We tried to use the poor energy resolution of the $H R I$ and derived a hardness ratio as defined by Huélamo et al. (2000). However, the gain of the $H R I$ was adjusted in December 1997, between our two observations. Therefore,

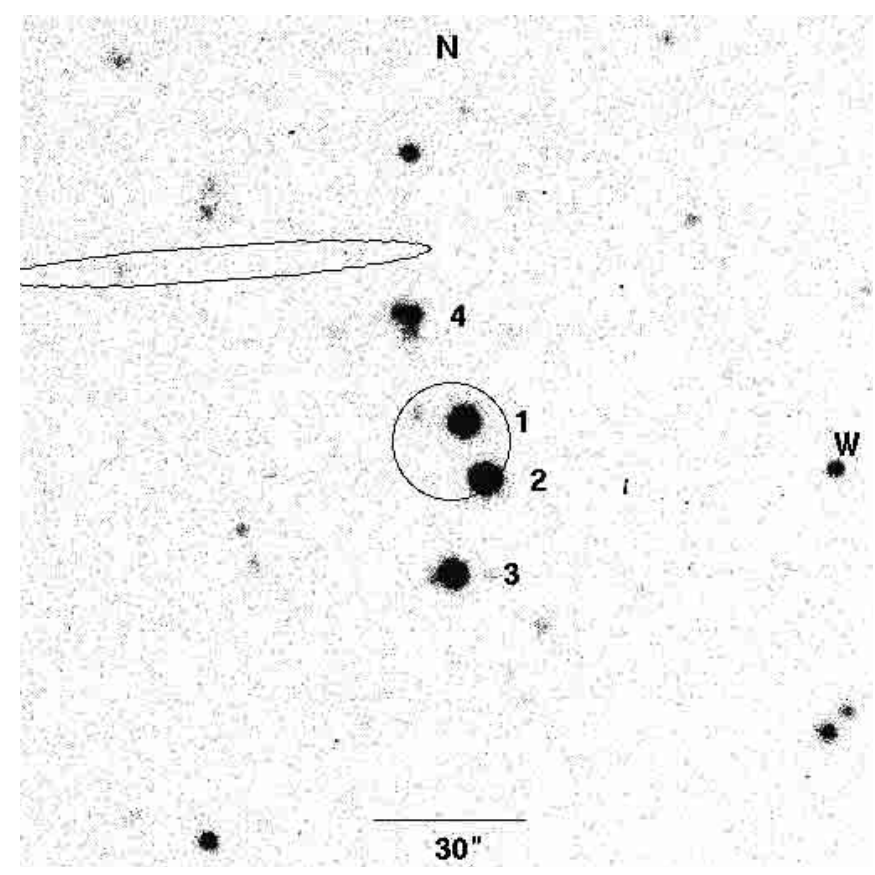

Fig. 2. $V$ image of surroundings of RX J2217.9-5941, source \#1. The center of the circle marks the RASS position and the radius is at the $90 \%$ confidence level. The positions and colours of the four sources marked here are given in Table 1. The IRAS position is marked by the $90 \%$ error ellipse in the upper left part of the figure

the systematic errors caused by this gain shift and gain adjustment are too large to retrieve meaningful results (e.g. Leighly et al. 1997).

Figure 3 displays the RASS lightcurve. Remarkably, the count rate decreased by a factor of $\approx 12$ in about two days. We could not detect any significant spectral change between the high and the low states due to the low photon statistics in the RASS data. A variability test of the RASS light curve gave a $\chi^{2} / \nu=4.2$ which makes RX J2217.95941 one of the most variable sources on short timescales throughout our complete sample of soft X-ray selected AGN (Grupe et al. 2001). A more common parameter often used in variability studies is the excess variance (e.g. Nandra et al. 1997; Leighly 1999a). The excess variance of RX J2217.9-5941 is $\sigma_{\text {rms }}^{2}=0.36 \pm 0.03$. In order to compare with the $A S C A$ results on NLS1s, we estimated the 2-10 keV rest-frame luminosity using a two component 
spectral fit to the RASS data assuming the ASCA spectral slope of $\alpha_{\mathrm{x}}=1.6$ (Sect. 3.2.2). We find that with the $2-10 \mathrm{keV}$ rest-frame luminosity $\log L_{2-10}=36.4$, RX J2217.9-5941 is one of the more variable NLS1 (Fig. 4, see also Fig. 3 in Leighly 1999a). Note that the length of the RASS observation is similar to that of the ASCA observations reported in Leighly (1999a), so that the comparison with the results reported in that paper is valid, but also note that in general the excess variance observed in hard X-rays is lower than observed in soft X-rays (e.g. Leighly 1999a). The HRI light curves were checked for variability as well. In both observations the result from the $\chi^{2}$ test is consistent with no variability.

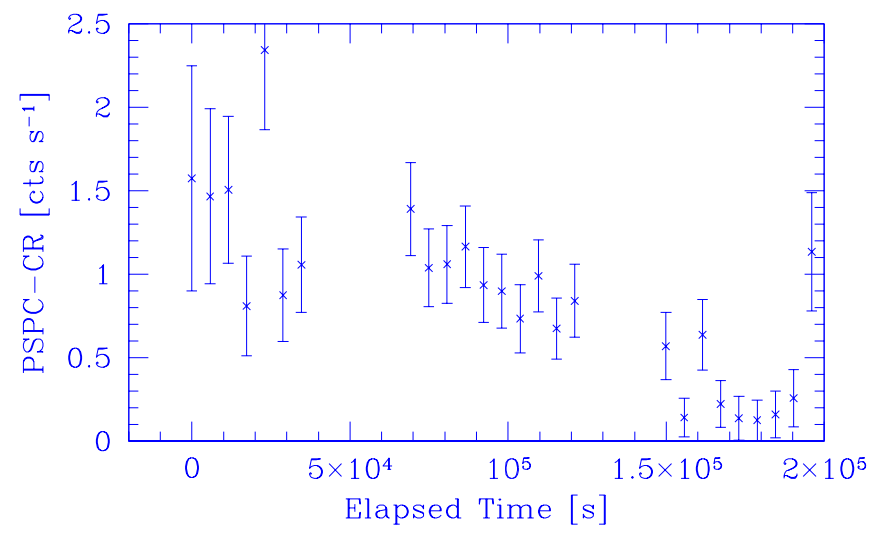

Fig. 3. Light curve of RX J2217.9-5941 during the RASS coverage

\subsubsection{ASCA observations}

Figure 5 shows the ASCA soft and hard band images generated from the SIS0 and SIS1 detectors. These have been adaptively smoothed, divided by an exposure map generated by the ascaexpo tool, and further smoothed with a boxcar average function 5 pixels wide. The " $\times$ " and "+" mark the HRI positions of RX J2217.9-5941 (source A in Table 2 and Fig. 1) and the bright unknown source RX J2217.9-5937 (source B), respectively. This demonstrates that the photometry in the ASCA image is adequate to identify the rather faint source as RX J2217.95937. RX J2217.9-5941 is clearly detected in the soft-band image but cannot be clearly identified in the hard band image.

Analysis of the ASCA data is complicated by the two sources only $4^{\prime}$ away. While the point-spread function of ASCA near the optical axis of the SIS has a relatively sharp core about 1 arcmin $F W H M$, it has broad wings (half-power diameter of $3 \mathrm{arcmin}$ ), and it is a function of energy. Thus the sources near RX J2217.9-5941 contaminate the spectrum drawn from a region including that source in a way that is non-negligible and difficult to estimate. It is possible to extract useful and unambiguous spectral information from faint sources located near bright sources by using the TRACE_ASCA software

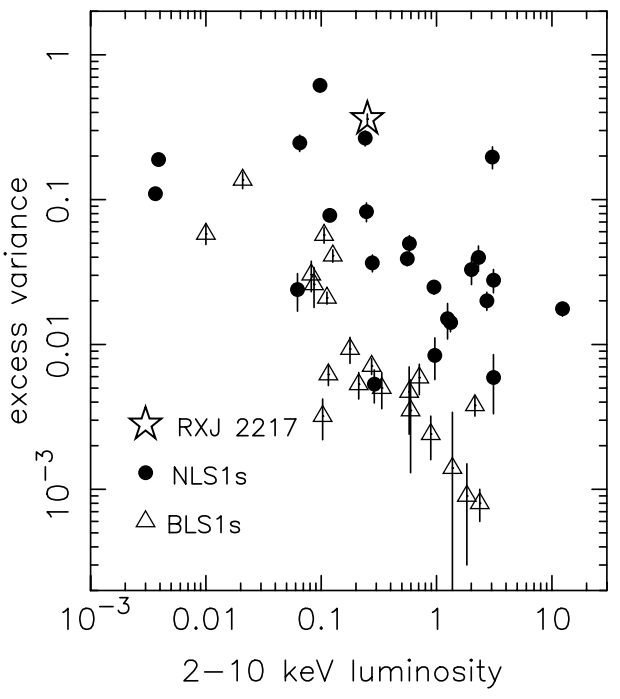

Fig. 4. Excess variance of RX J2217.9-5941 vs. 2-10 keV restframe luminosity in comparison with the sample of Leighly (1999a,b). The $2-10 \mathrm{keV}$ rest-frame luminosity is given in units of $10^{37}$ Watts

developed by A. Ptak to simulate the contribution of the bright source in the extraction region of the fainter source. However, we do not employ this rather complex analysis technique here simply because the statistics are rather poor and are likely to dominate the spectral analysis. The GIS detector smears the X-ray image more and therefore we do not consider the GIS data at all here.

We finally decided to extract and analyze spectra from a region obtained within a 1.5 arcmin radius region. This region size is much smaller than the nominal SIS extraction size of 4 arcmin radius; however, the proximity of nearby sources required small region sizes so that the regions would not overlap and so that the contamination of the flux from the neighboring sources would be minimized. Significant source photons will be outside of these small region sizes; however, the ancillary response files account for the reduction in effective area and thus the measured flux would not be affected. In this case, however, the measured flux will be an upper estimate because of the contamination by neighboring sources. The ASCA PSF is energy dependent and hard photons are scattered more than soft photons. This effect is also accounted for by the ancillary response file, except for the fact that the high energy spectrum will be less well constrained than it would be if the nominal region size could be used.

We first attempted to determine whether the sources were variable. Light curves binned by orbit were extracted and examined, and no clearly convincing evidence of variability was found during the observation in any of the three sources. Statistical evaluation of the variability was hampered by the low count rates: the number of photons per orbit was so small that the uncertainties should be governed by Poisson rather than Gaussian statistics and thus the errors were not trivially evaluated. Maximum likelihood techniques can be applied when the count rates 

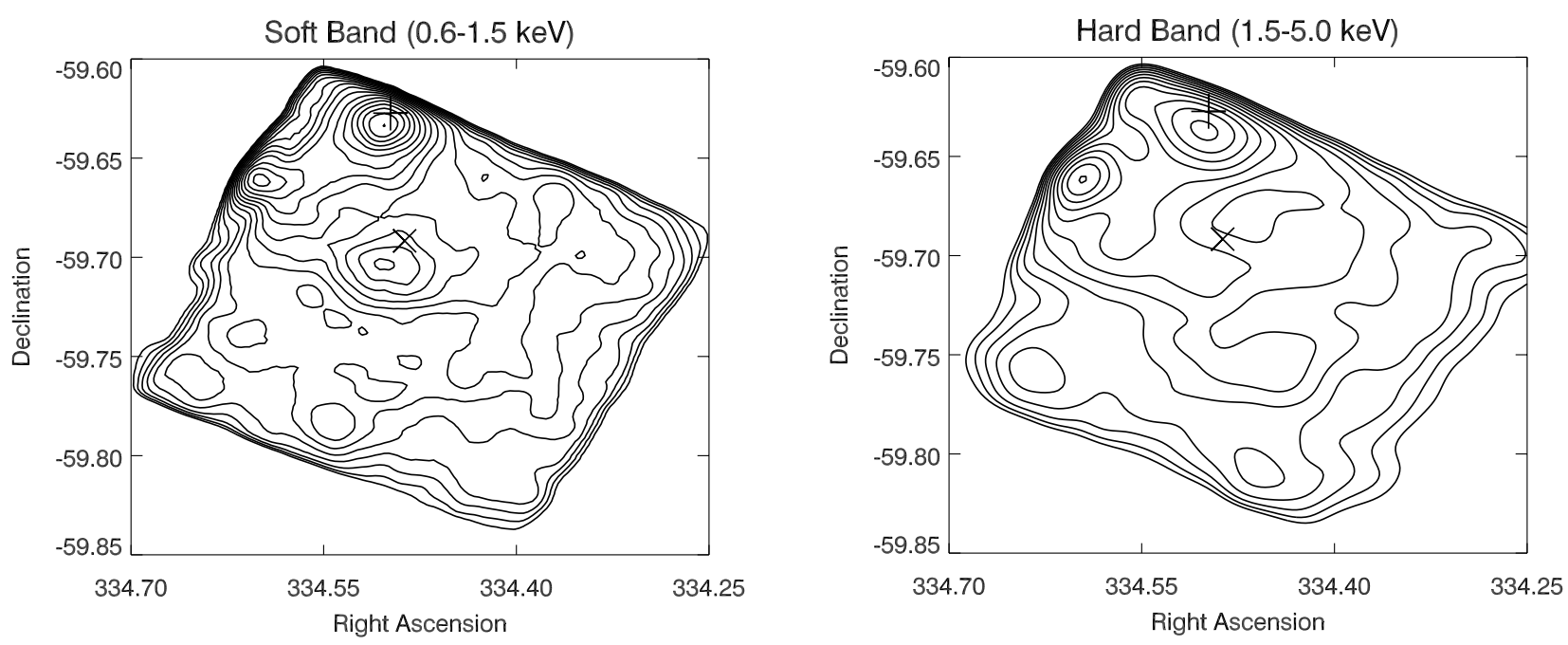

Fig. 5. ASCA image of RX J2217.9-5941. The HRI position of RX J2217.9-5941 (source A in Table 2 and Fig. 1) is marked by an " $\times$ " and the HRI position of the bright source RX J2217.9-5137 (source B) by a "+". Note that RX J2217.9-5941 is only clearly detected in the soft band image, a fact that supports the steep spectrum derived from spectral analysis. $H R I$ positions for both sources are given in Sect. 3.1

are low (e.g. Leighly 1999a); however, these techniques must be used on counts light curves rather than rate light curves, and counts light curves could not be constructed because there was a wide distribution of orbital net exposures.

Table 2 gives the results of spectral fitting. Statistics were poor enough that the only model used to fit the spectra was a power law with the column density fixed at the Galactic value. It is noteworthy that the best fitting spectral index of RX J2217.9-5941 is $\alpha_{\mathrm{x}}=1.6$; this is the typical maximum value obtained from other NLS1s (e.g. Leighly 1999b). We note that because of the source contamination and because of the small number of photons, our derived ASCA spectral index must be considered extremely tenative. However, it seems fairly clear that the spectrum of the object is not flat as it would be if the spectrum were either dominated by reflection $\left(\alpha_{\mathrm{X}} \leq 0\right.$; e.g. Matt et al. 1996) or absorbed. The fact that the source is detected clearly only in the soft band image also supports a soft spectrum.

The identity of the two other objects in the ASCA field of view is unknown. The HRI astrometry is good enough that RX J2217.9-5937 (source B) can be fairly confidently identified with an object appearing in the Digitized Sky Survey at $221759.2,-593741$ (J2000). Although the USNO catalog magnitudes are only reliable within about $0.5 \mathrm{mag}$, we can use them to get an idea of the identity of the object. The USNO catalog lists the $R$ and $B$ band magnitudes of this source as 18.0 and 18.7 respectively. Assuming that the spectrum is a power law gives an estimate of the $V$ band magnitude of 18.4. The best fitting model of the ASCA spectra gives an estimated flux in the $0.3-3.5 \mathrm{keV}$ band of $1.510^{-17} \mathrm{~W} \mathrm{~m}^{-2}$. Comparison of these values with the X-ray source nomogram (Maccacaro et al. 1988), and assuming no intrinsic absorption or reddening, shows that the optical and X-ray properties of the object are consistent with it being an AGN.

Identification of the optical object associated with RX J2218.0-5939 (source C) is more difficult because it is a weaker object. There is an optical source within 7 arcsec identified in the USNO catalog as having $R$ and $B$ mag of 18.3 and 20.9 respectively.

\subsection{Optical}

The optical spectrum of RX J2217.9-5142 (Fig. 6) shows the typical spectrum of a Narrow-Line Seyfert 1 galaxy. The full width of the $\mathrm{H} \beta$ line was measured to $1850 \pm$ $100 \mathrm{~km} \mathrm{~s}^{-1}$. The spectrum appears to be unreddened $\left(\mathrm{H} \alpha / \mathrm{H} \beta=3.4, \alpha_{\mathrm{opt}}=0.3\right.$; Grupe et al. 1999a). We subtracted an FeII template from all optical spectra of RX J2217.9-5941 (see e.g. Boroson \& Green 1992; Grupe et al. 1999a).

The redshift of RX J2217.9-5941 based on the $\mathrm{H} \alpha$ and $\mathrm{H} \beta$ lines was $z=0.160$ (Grupe et al. 1998a, 1999a). The [OIII] lines show a blueshift of about $570 \pm 20 \mathrm{~km} \mathrm{~s}^{-1}$ with respect to the Balmer lines; this will be discussed further in Grupe \& Leighly (in prep.). The line width of the [OIII] lines is extremely broad compared to the rest of the sample. We measured a $F W H M([\mathrm{OIII}] \lambda 5007)=$ $1075 \pm 150 \mathrm{~km} \mathrm{~s}^{-1}$ (Grupe et al. 1999a).

Erkens et al. (1997) reported a correlation between the strength of coronal iron lines and the steepness of the ROSAT spectra in AGN. Because RX J2217.9-5941 has a very steep X-ray spectrum, we looked for coronal iron lines but did not find any in any of the spectra. A comparison of the spectra taken in 1992, 1993, 1995, and 1998 does not show detectable changes in line and continuum fluxes. 
Table 2. Power-law fits to the ASCA spectra of the objects listed below. The absorption parameter $N_{\mathrm{H}}$ was fixed to the galactic value (see text). The $2-10 \mathrm{keV}$ model flux is given in units of $10^{-17} \mathrm{~W} \mathrm{~m}^{-2}$, the predicted and measured $H R I$ count rates are in units of $10^{-3} \mathrm{cts} \mathrm{s}^{-1}$

\begin{tabular}{clcrrrrrr}
\hline Source & Object $^{a}$ & $\begin{array}{c}\text { total \# } \\
\text { of photons }^{b}\end{array}$ & $\alpha_{\mathrm{X}}$ & $\begin{array}{r}2-10 \mathrm{keV} \\
\text { Flux }^{c}\end{array}$ & $\chi^{2} /$ dof & $\begin{array}{c}\text { HRI predicted } \\
\text { count rate }^{d}\end{array}$ & \multicolumn{2}{c}{ HRI measured CR } \\
1997 & 1998 \\
\hline A & RX J2217.9-5941 & 187 & $1.60_{-0.58}^{+0.66}$ & $2.7_{-1.6}^{+2.5}$ & $6.2 / 11$ & $2.88_{-1.10}^{+1.54}$ & $51.9 \pm 4.50$ & $5.40 \pm 1.80$ \\
B & RX J2217.9-5937 & 284 & $1.09_{-0.29}^{+0.32}$ & $9.7_{-3.2}^{+3.5}$ & $21.8 / 20$ & $3.92_{-0.88}^{+0.96}$ & $8.37 \pm 2.36$ & $4.36 \pm 1.24$ \\
C & RX J2218.0-5939 & 126 & $0.69_{-0.52}^{+0.52}$ & $13.2_{-6.3}^{+9.0}$ & $5.5 / 6$ & $2.65_{-0.86}^{+1.10}$ & - & - \\
\hline
\end{tabular}

$a$ The exact $H R I$ positions of these sources are given in Sect. 3.1.

${ }^{b}$ Total number of photons in the extraction regions for SIS0+SIS1 between 0.6 and $5 \mathrm{keV}$. This includes source photons, background photons, and photons from the PSF wings of the other sources. The number of contributing background photons is estimated to be between 80 and 100 counts in the same energy band for two detectors.

${ }^{c}$ Flux uncertainties were generated by determining fluxes for values of photon index and normalization separately that produced worse fits by $\Delta \chi^{2}$ of 2.71 .

${ }^{d}$ Based on spectral fits to the ASCA data and usage of the response matrix.

${ }^{e}$ Note that RX J2218.0-5939 was observed only in the SIS0 detector.

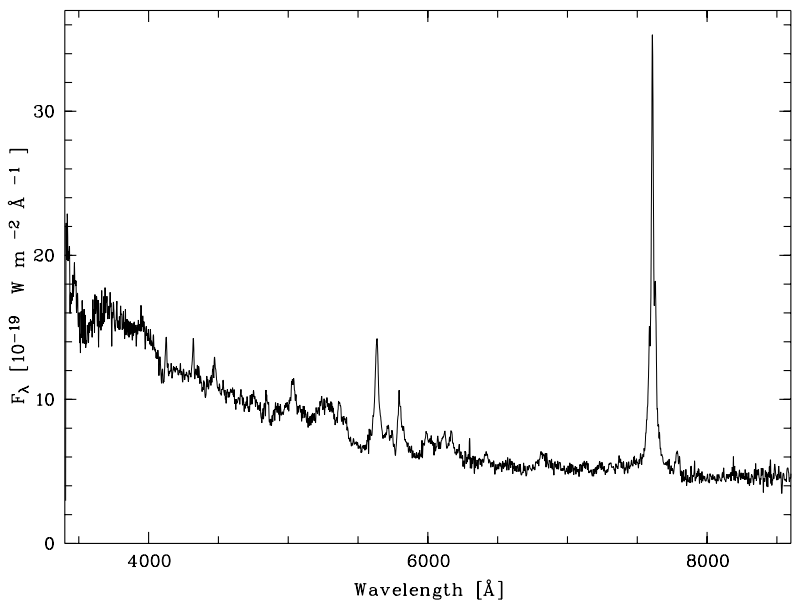

Fig. 6. Optical spectrum of RX J2217.9-5941 obtained with the $1.5 \mathrm{~m}$ telescope at CTIO

\subsection{Other wavelength ranges}

Infrared: RX J2217.9-5841 is listed as a bright infrared IRAS source in our soft X-ray selected AGN sample, and it is also listed as in infrared source in the samples of Boller et al. (1992, 1997b) and Moran et al. (1996). However, a comparison of the IRAS and ROSAT positions reveals that RX J2217.9-5941 is probably not the infrared source. The IRAS position marked in Fig. 2 is $\alpha_{2000}=22^{\mathrm{h}} 18^{\mathrm{m}} 03.3$ and $\delta_{2000}=-59^{\circ} 40^{\prime} 58^{\prime \prime}$ (Boller et al. 1997b). So either the faint object $10^{\prime \prime}$ north of the IRAS position or object \#4 in Fig. 2 is the optical counterpart of the IRAS source.

Radio: We have searched for radio data for RX J2217.95941. The nearest entry in the Parkes-MIT-NRAO survey catalog (PMN, Wright et al. 1994; Condon et al. 1998) is at $\alpha_{2000}=22^{\mathrm{h}} 17^{\mathrm{m}} 28.1^{\mathrm{s}}, \delta_{2000}=-59^{\circ} 31^{\prime} 10.0^{\prime \prime}$, about $11^{\prime}$ away from the position of our source, and too far away to be associated with RX J2217.9-5941.

\subsection{Spectral energy distribution}

In order to obtain an estimate of the bolometric luminosity, which is used later to obtain a limit on the black hole mass, we performed a two-component spectral fit to the optical and RASS X-ray data consisting of a lowenergy power law with exponential cut-off and a highenergy power law. The low-energy part represents a simple geometrically thin accretion disk model in LTE (e.g. Krolik 1999), with $\alpha=-1 / 3$. The optical spectrum (see Fig. 6) was corrected for interstellar extinction using the relation between $E_{B-V}$ and Galactic column density given by Diplas \& Savage (1994), a value of $A_{V} / E_{B-V}=3.2$ (Seaton 1979), and the wavelength dependence of $A_{\lambda}$ derived by Cardelli et al. (1989). At the blue end of the optical spectrum $\left(\nu=8.410^{14} \mathrm{~Hz}\right)$, where the contribution of the underlying galaxy is small, we obtained a flux of $\nu F_{\nu}=7.4010^{-15} \mathrm{~W} \mathrm{~m}^{-2}$. We fixed the column density $N_{\mathrm{H}}$ to the Galactic value and the hard X-ray spectral slope to $\alpha_{\mathrm{x}}=1.6$, the value derived from the ASCA data (see Sect. 3.2.2 and Table 2). The fit to the $\mathrm{X}$-ray data then gave a cut-off energy of $41.9 \pm 1.2 \mathrm{eV}$, and a normalisation for the high-energy power law of $\nu F_{\nu}=$ $(1.06 \pm 0.26) 10^{-15} \mathrm{~W} \mathrm{~m}^{-2}$ at $1 \mathrm{keV}$, with $\chi^{2} / \nu=10.3 / 9$. The Wien tail of the low-energy component (in the $\mathrm{X}$ ray regime) corresponds to a power law with $\alpha_{\mathrm{x}}=4.4$. Figure 7 displays the result of this fit. Although the optical spectrum obtained in 1998 was not simultaneous with the RASS data, $V$ mag obtained in Aug. 92 and Sep. 93 differ by 0.08 mags only. The total luminosity over the optical to X-ray range, where most of the energy is radiated, is $\log L=39.1[\mathrm{~W}]$.

Based on the model described above we estimated the rest-frame fluxes at $2500 \AA$ and $2.0 \mathrm{keV}$ in order to derive the optical/UV to X-ray slope $\alpha_{\mathrm{ox}}$. For the RASS observation we found $\alpha_{\mathrm{ox}}=1.52$, and for the two pointed $H R I$ observations in 1997 and 1998 we derived $\alpha_{\mathrm{ox}}=1.62$ and $\alpha_{\mathrm{ox}}=2.00$, respectively, assuming that the spectral shape had not changed and that the optical flux at $2500 \AA$ 


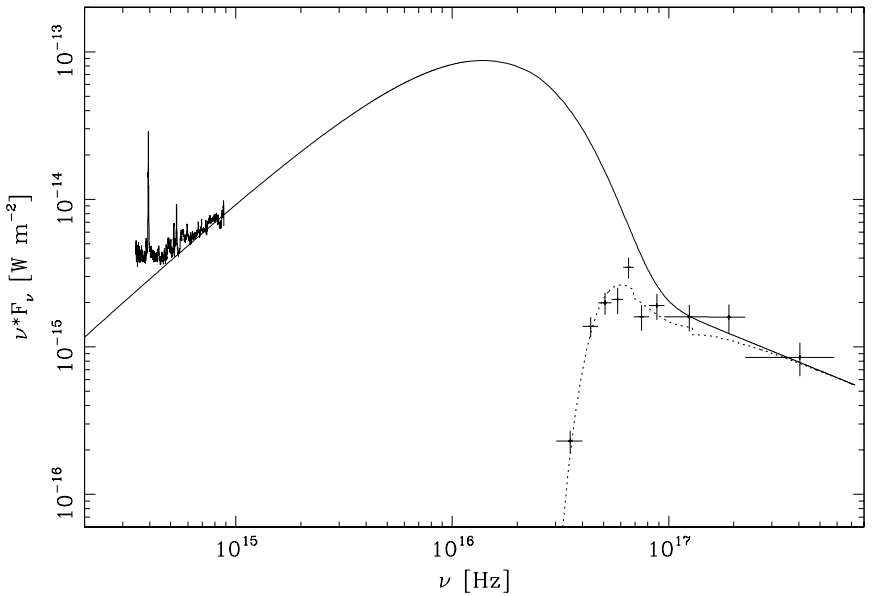

Fig. 7. Optical to X-ray spectrum of RX J2217.9-5941. The solid line shows the model as described in Sect. 3.5, the dashed line the absorbed spectrum fitted to the RASS spectrum (marked by crosses)

was constant. This is in the range reported for the PG quasar sample of Tananbaum et al. (1986) by Wilkes et al. (1994; see their Fig. 6b). Following Eq. (12) in Wilkes et al. (1994), from the luminosity at $2500 \AA$ an $\alpha_{\text {ox }}=1.47$ would be expected. Here a $H_{0}=50 \mathrm{~km} \mathrm{~s}^{-1} \mathrm{Mpc}^{-1}$ was used to be consistent with Wilkes et al. (1994). This is in agreement with the RASS value. However, the second HRI and the ASCA observations lay at the very steep end compared to the PG quasar sample. We also compared our values with the larger data base for radio-quiet quasars compiled by Yuan et al. (1998). They find a mean $\alpha_{\text {ox }}$ of $1.51 \pm 0.03$ for the redshift and luminosity range of RX J2217.9-5941. This is precisely the value we found for the RASS observation, but again much smaller than the values we obtained from the HRI and ASCA observations.

We also checked $\log \left(f_{\mathrm{x}} / f_{\mathrm{O}}\right)$ as given in Eq. (2) in Beuermann et al. (1999) and compared it with their Fig. 2. We found that the RASS as well as the first $H R I$ pointing still fall into the region where AGN are expected. The second $H R I$ pointing is, considering the spectrum is still steep, way off the AGN region $\left(\log \left(f_{\mathrm{x}} / f_{\mathrm{o}}\right)=+0.54,+0.03\right.$, and -0.97 for the RASS, the $1997 H R I$ and the $1998 H R I$ observations, respectively).

\section{Discussion}

RX J2217.9-5941 is one of the most variable AGN on short timescales of days to long timescales of years in our sample of soft X-ray selected AGN (Grupe et al. 2001). The variability of RX J2217.9-5941 shows:

1. a strong decrease in PSPC count rate in its "high" state during the RASS, but no significant variability during the three observed "low" states during the pointed ROSAT and ASCA observations;

2. an apparent slow decrease in count rate/flux on long time scales.
In principle, variability can have two origins: either it is intrinsic or it results from changes of the cold or warm absorption column densities. In the first case, the variability originates in the AGN engine itself, and it may be either persistent or a transient. In the second case, absorbing clouds are in the line-of-sight. We now discuss the possibilities of a transient event or persistent variability in turn.

\subsection{Transient event?}

Some results seem to argue for a transient nature of RX J2217.9-5941:

1. During three pointings after the RASS the source was seen in "low" state;

2. From the $H R I$ pointing in 1997 to the $H R I$ observation in 1998 the source decreased in count rate by a factor of about 10 and even the ASCA observation three weeks after the $1998 H R I$ observation is fainter by about a factor of 2 (see Table 2);

3. The X-ray spectrum is steep. All transients discovered so far showed very steep X-ray spectra.

We can imagine two forms of X-ray outburst events, either an instability in the accretion disk that causes an dramatic increase in the accretion rate, or a tidal disruption of a star by the central black hole. An instability in the accretion disk can cause a higher accretion rate over a short time or a decrease in the accretion rate. In both cases we would expect to see a decrease in the X-ray flux over long time-scales. This can be a one-time event, but it can be also persistent variability. Tidal disruption of a star by a central black hole appears when the path of a star comes too close to the central black hole and gets disrupted by the gravitational force (Rees 1990; Ayal et al. 2000). This model can explain X-ray outbursts in nonactive galaxies such as RX J1624.9+7554 (Grupe et al. 1999b) or the Seyfert 2 galaxy IC 3599 (Brandt et al. 1995; Grupe et al. 1995). However, all those objects are inferred to have black hole masses in the order of $10^{6} M_{\odot}$. The black hole in RX J2217.9-5941 is about two orders of magnitude larger. We can estimate the lower limit of the black hole mass by assuming that the highest luminosity seen in this source is the Eddington luminosity. With a mean bolometric luminosity of $\log L_{\mathrm{bol}}=39.1$ [W] derived from the RASS and the optical observations (see Sect. 3.2.1) we get a minimum black hole mass of RX J2217.9-5941 of $\approx 10^{8} M_{\odot}$. Even if it is not impossible for a $10^{8} M_{\odot}$ black hole to disrupt a star, it is less likely simply due to the size of the black hole. The star must cross the region between the event horizon and the tidal disruption radius in order for us to see a transient event. This region becomes smaller as the BH mass increases because the radius of the event horizon scales with the mass of the black hole while the tidal disruption radius scales with $M^{1 / 3}$ (Rees 1990). 


\subsection{Persistent variability?}

The variable X-ray source could be the accretion disk itself. A cut-off powerlaw, which has a shape of a simple accretion disk model, fits the observed data in the optical and X-ray part of the spectrum quite well. Thus the $\mathrm{X}$-rays are emitted from the inner part of the disk (3$10 R_{\mathrm{S}}$ ), while the optical emission is produced further out. The bolometric luminosity of $\log L_{\mathrm{bol}}=39.1$ observed in RX J2217.9-5941 requires an accretion rate of $1.4 M_{\odot} \mathrm{y}^{-1}$ assuming an efficiency of $\eta=0.1\left(L=\eta \dot{M} \mathrm{c}^{2}\right)$. This high accretion rate might explain the strong decrease in count rate during the "high" state RASS observation while during the "normal" low states in later pointings the accretion rate is more stable. That is, during the RASS, the source may have been accreting close to the Eddington limit, giving a very soft X-ray spectrum (e.g. Pounds et al. 1995; Ross et al. 1992). In later years, the accretion rate decreased, resulting in a lower temperature in the inner part of the disk and shifting the soft X-ray component out of the ROSAT window; this explanation was also proposed for the X-ray transient WPVS007 (Grupe et al. 1995b).

However, it can not be the soft X-ray part alone that must have changed. During the RASS, the contribution to the total count rate from the hard X-ray spectrum alone was $0.28 \mathrm{cts} \mathrm{s}^{-1}$, assuming $\alpha_{\mathrm{x}}=1.6$. Assuming that during the pointed observations all of the soft component flux was gone, the $H R I$ count rate predicts $0.155 \mathrm{PSPC} \mathrm{cts} \mathrm{s}^{-1}$ for the $1997 \mathrm{HRI}$ observation and 0.016 PSPC $\mathrm{cts} \mathrm{s}^{-1}$ (with $\alpha_{\mathrm{x}}=1.6$ and $N_{\mathrm{H}}=$ galactic).

An alternative explanation for persistent variability is relativistic Doppler boosting. We cannot completely exclude this explanation, however, the boosting factor is very sensitive to the inclination angle and strongest when the disk is viewed edge-on. RX J2217.9-5941 is a NLS1 and these sources are usually thought to be viewed at low inclination angles (e.g. Grupe et al. 1998b) and therefore the boosting factor should be low. Nevertheless, Boller et al. (1997) suggested relativistic Doppler boosting to explain the rapid and persistent variability in the NLS1 IRAS 13224-3809. However, we observe strongly blueshifted broad high-ionization lines with nearly no emission redward of the rest wavelength, while the low ionization lines are narrow and symmetric. A disk-wind model most naturally explains these results, and therefore an edge-on orientation is very unlikely (Leighly \& Halpern 2001; Leighly 2000).

Also arguing for a non-transient nature of RX J2217.9 5941 is the optical-to-X-ray slope $\alpha_{\text {ox }}$. During the RASS it was in the range found for quasars by Yuan et al. (1998), Wilkes et al. (1994), and Brandt et al. (2000), and agrees with the expected value of $\alpha_{\mathrm{ox}}$ derived from the optical/UV luminosity as defined by Wilkes et al. (1994). The slope $\alpha_{\mathrm{ox}}$ derived from the HRI and ASCA observations are much steeper compared to those samples, indicating that RX J2217.9-5941 is deficient in X-rays. Brandt et al. (2000) have investigated "soft X-ray weak" AGN and find a clear correlation between the CIV absorption line equivalent width and $\alpha_{\text {ox }}$, suggesting that soft X-ray weak objects are absorbed (also Schartel et al. 1997). There was certainly no X-ray absorption present during the RASS observation (when the $\alpha_{\mathrm{ox}}$ was in the normal range). Although the ASCA results are extremely tentative because of poor statistics and contamination, we find no evidence to suggest that the X-ray spectrum is absorbed when RX J2217.9-5941 was in the faint state. However, we will have to wait for follow-up Chandra or XMM observations to make any definitive statements about the shape of the X-ray spectrum and whether or not there is any X-ray absorption present. HST observations would reveal whether there are deep UV absorption lines present.

Another argument for persistent variability in RX J2217.9-5941 is that strong variability is common among NLS1 (Leighly 1999a,b) and RX J2217.9-5941 is a typical NLS1.

Another possibility is that we are looking at two kinds of variability. Absorption could have been responsible for the short-time variability during the RASS when the source was in its "high" state, when there was a strong decrease with no evidence for a change in spectral shape (although due to the poor signal to noise, we cannot place very strong constraints on the spectral variability). If there were no spectral variability, the source flux variability could have been caused by a cold absorbing cloud moving over the a period of two days into the line of sight between us and the X-ray source. If the central region is filled with patchy clouds, they can indeed cause variability and has been proposed by several workers (e.g. Abrassart \& Czerny 2000; Schartel et al. 1997; Komossa \& Meerschweinchen 2000).

\section{Summary and conclusions}

1. RX J2217.9-5941 has shown an remarkable decrease in count rate by a factor of about 40 between the RASS and pointed observations 8 years later;

2. During the 2 day RASS coverage the count rate decreased by a factor of 15 ;

3. The optical/UV to X-ray spectral slope $\alpha_{\mathrm{ox}}$ and the flux ratio $f_{\mathrm{X}} / f_{\mathrm{O}}$ argue more for a persistent variability than a one time outburst event;

4. In case of an outburst event, tidal disruption of a star by the central black hole is most unlikely due to the mass of the black hole of at least $10^{8} M_{\odot}$;

5 . The $[\mathrm{OIII}]$ line system is blueshifted with respect to the $\mathrm{H} \beta$ line and the [OIII] emission lines are broader $\left(F W H M([\mathrm{OIII}])=1075 \mathrm{~km} \mathrm{~s}^{-1}\right)$ than the $\mathrm{H} \beta$ emission line;

6. The only way to see if RX J2217.9-5941 is a transient source with a constantly decreasing count rate or if it is indeed persistent variability, is to monitor this source with new X-ray missions like XMM and Chandra. With XMM and its Optical Monitor we would even be able to observe in the optical/UV and $\mathrm{X}$-ray ranges simultaneously; 
7. A possible check to estimate the "normal" $\alpha_{\mathrm{ox}}$ is to observe the CIV absorption line in the UV.

Acknowledgements. We would like to thank Prof. Jules Halpern for taking the optical spectrum of RX J2217.9-5941 at CTIO in 1998 and Dr. Klaus Reinsch for observing source \#2 (see Table 1) at ESO in January 2001. We thank Drs. Wolfgang Brinkmann and Mario Gliozzi for their comments and suggestion on the manuscript. This research has made use of the NASA/IPAC Extragalactic Database (NED) which is operated by the Jet Propulsion Laboratory, Caltech, under contract with the National Aeronautics and Space Administration. We also used the IRAS data request of the Infrared Processing and Analysis Center (IPAC), Caltech. KML gratefully acknowledges support through NAG5-7971 (NASA, LTSA). The ROSAT project is supported by the Bundesministerium für Bildung und Forschung (BMBF/DLR) and the Max-PlanckSociety

This paper can be retrieved via WWW:

http://www . xray.mpe.mpg. de/ dgrupe/research/refereed.html

\section{References}

Abrassart, A., \& Czerny, B. 2000, A\&A, 356, 475

Ayal, S., Livio, M., \& Piran, T. 2000, ApJ, 545, 772

Beuermann, K., Thomas, H.-C., Reinsch, K., et al. 1999, A\&A, 347,47

Boller, Th., Meurs, E. J. A., Brinkmann, W., et al. 1992, A\&A, 261,57

Boller, Th, Brandt, W. N., \& Fink, H. H. 1996, A\&A, 305, 53

Boller, Th., Brandt, W. N., Fabian, A. C., \& Fink, H. H. 1997a, MNRAS, 289, 393

Boller, Th., Bertoldi, F., Dennefeld, M., \& Voges, W. 1997b, A\&AS, 129, 87

Boroson, T. A., \& Green, R. F. 1992, ApJS, 80, 109

Brandt, W. N., Pounds, K. A., \& Fink, H. H. 1995, MNRAS, 273, L47

Brandt, W. N., Boller, Th., Fabian, A. C., \& Ruszkowski, M. 1999, MNRAS, 303, L53

Brandt, W. N., Laor, A., \& Wills, B. J. 2000, ApJ, 528, 637

Condon, J. J., Cotton, W. D., Greisen, E. W., et al. 1998, AJ, 115,1693

Dickey, J. M., \& Lockman, F. J. 1990, ARA\&A, 28, 215

Elvis, M., Giommi, P., Wilkes, B. J., \& McDowell, J. 1991, ApJ, 378, 537

Erkens, U., Appenzeller, I., \& Wagner, S. 1997, A\&A, 323, 707

Frank, J., King, A. R., \& Raine, D. J. 1992, Accretion power in astrophysics (Cambridge UK, University Press), 2nd edition

Forster, K., \& Halpern, J. P. 1996, ApJ, 468, 565

Greiner, J., Schwarz, R., Zharikov, S., \& Orio, M. 2000, A\&A, $362, \mathrm{~L} 25$

Grupe, D. 1996, Ph.D. Thesis, University of Göttingen

Grupe, D., Beuermann, K., Mannheim, K., et al. 1995a, A\&A, 299, L5

Grupe, D., Beuermann, K., Mannheim, K., et al. 1995b, A\&A, 300, L21

Grupe, D., Beuermann, K., Mannheim, K., Thomas, H.-C., \& Fink, H. H. 1998a, A\&A, 330, 25
Grupe, D., Wills, B. J., Wills, D., \& Beuermann, K. 1998b, A\&A, 333, 827

Grupe, D., Beuermann, K., Mannheim, K., \& Thomas, H.-C. 1999a, A\&A, 350, 805

Grupe, D., Thomas, H.-C., \& Leighly, K. M. 1999b, A\&A, 350, L31

Grupe, D., Leighly, K. M., Thomas, H.-C., \& LaurentMuehleisen, S. A. 2000, A\&A, 356, 11

Grupe, D., Thomas, H.-C., \& Beuermann, K. 2001, A\&A, in press

Guainazzi, M., Nicastro, F., Fiore, F., et al. 1998, MNRAS, 301, L1

Huélamo, N., Neuhäuser, R., Stelzer, B., Supper, R., \& Zinnecker, H. 2000, A\&A, 359, 227

Komossa, S., \& Greiner, J. 1999, A\&A, 349, L45

Komossa, S., \& Meerschweinchen, J. 2000, A\&A, 354, 411

Krolik, J. H. 1999, in Theory of black hole Accretion Disks, ed. M. A. Abramowicz, G. Björnssson, \& J. E. Pringle (Cambridge University Press), 134

Leighly, K. M. 2000, Proc. "Observational and Theoretical Progress in the Study of Narrow-line Seyfert 1 Galaxies", ed. T. Boller, W. N. Brandt, K. M. Leighly, \& M. Ward, New Astron. Rev., 44, 395

Leighly, K. M. 1999a, ApJS, 125, 297

Leighly, K. M. 1999b, ApJS, 125, 317

Leighly, K. M. 2000, ApJ, submitted

Leighly, K. M., \& Halpern, J. P. 2001, ApJ, submitted

Leighly, K. M., O'Brian, P. T., Edelson, R., et al. 1997, ApJ, 483, 767

Maccacaro, et al. 1988, ApJ, 326, 680

Matt, G., Fiore, F., \& Perola, G. C. 1996, MNRAS, 281, L69

Moran, E. C., Halpern, J. P., \& Helfand, D. J. 1996, ApJS, 106, 341

Nandra, K., George, I. M., Mushutzky, R. F., Turner, T. J., \& Yaqoob, T. 1997, ApJ, 476, 70

Pfeffermann, E., Briel, U. G., \& Hippmann, H. 1986, SPIE, 733,519

Pounds, K. A., Done, C., \& Osborne, J. P. 1995, MNRAS, 277, L5

Rees, M. J. 1990, Science, 247, 817

Ross, R. R., Fabian, A. C., \& Mineshige, S. 1992, MNRAS, 258,189

Schartel, N., Komossa, S., Brinkmann, W., et al. 1997, A\&A, 320,421

Seaton, M. J. 1979, MNRAS, 187, 73P

Thomas, H.-C., Beuermann, K., Reinsch, K., et al. 1998, A\&A, 335,467

Trümper, J. 1983, Adv. Space Res., 4, 241

Voges, W., Aschenbach, B., Boller, Th., et al. 1999, A\&A, 349, 389

Wilkes, B. J., Tananbaum, H., Worrall, D. M., et al. 1994, ApJS, 92, 53

Wright, A. E., Griffith, M. R., Burke, B. F., \& Ekers, R. D. 1994, ApJS, 91, 111

Yuan, W., Brinkmann, W., Siebert, J., \& Voges, W. 1998, A\&A, 330, 108

Zimmermann, H. U. G., Boese, G. W., Becker, W., et al. 1998, EXSAS User's Guide, MPE Report 